\title{
STARLET: Multi-document Summarization of Service and Product Reviews with Balanced Rating Distributions
}

\author{
Giuseppe Di Fabbrizio*†, Ahmet Aker*, Robert Gaizauskas* \\ * Department of Computer Science \\ University of Sheffield, Sheffield, S1 4DP - UK \\ Email: \{G.DiFabbrizio,A.Aker,R.Gaizauskas\}@sheffield.ac.uk \\ $\dagger$ AT\&T Labs - Research \\ Florham Park, NJ 07932 - USA \\ Email:pino@research.att.com
}

\begin{abstract}
Reviews about products and services are abundantly available online. However, selecting information relevant to a potential buyer involves a significant amount of time reading user's reviews and weeding out comments unrelated to the important aspects of the reviewed entity. In this work, we present Starlet, a novel approach to multi-document summarization for evaluative text that considers the rating distribution as summarization feature to consistently preserve the overall opinions distribution expressed in the original reviews. We demonstrate how this method improves traditional summarization techniques and leads to more readable summaries.
\end{abstract}

Keywords-Summarization; evaluative text; $A^{*}$ search; multiratings prediction

\section{INTRODUCTION}

With the broad availability of always-connected portable devices such as mobiles, tablets, and eReaders, condensing information in a relatively small screen has become a necessity for the excitingly demanding population of users onthe-go. Retail industry and service providers are recognizing that there is a growing crowd of potential customers who are relying on their devices to learn about products and services, discover other user's experiences, and, ultimately, make a decision about spending their money or not [1], [2].

Although reviews about products and services are abundantly available online, selecting information relevant to a potential buyer involves a significant amount of time reading reviews and weeding out comments unrelated to the important aspects of the reviewed entity. This is particularly true for mobile users where additional constraints about geographic location together with limited visualization real estate may affect consumer's purchase behavior. In order to summarize reviews on mobile devices, several steps are required, each one involving different and often poorly integrated technology.

For instance, a first step in such a process would involve the use of local mobile search techniques [3] to find services or products available nearby. In this case, the search engine will have to re-rank output results by using geographic information about the current user's location [4] - or an explicitly requested location - and, optionally, re-score the final results based on previous search history captured in the user profile. Next, the user will start evaluating the search results by exploring reviews and ratings posted online by other users. In this case, opinion mining and sentiment analysis methods can be applied to extract the target of the opinions expressed in the reviews and the relative polarity (e.g., positive, negative, or neutral) [5], [6], [7]. Lastly, the user will be engaged in a complex task to process all the facts, opinions, and ratings read in the previous step and subsequently interpret, compare, contrast, and, finally, summarize the needed information.

While the first two steps have been largely explored, summarization of evaluative text (e.g., documents containing opinion or sentiment-laden text), is fairly new and may be substantially different from the traditional summarization task. In fact, most summarization techniques are focusing on distilling factual information by identifying the documents main topics, removing redundancies, and coherently ordering the extracted phrases or sentences. Most of the contributions have been developed using corpora with well-formed documents from domains such as news articles [8], [9], medical literature [10], biographies [11], technical articles [12], and blogs [13]. As observed in Ku et al. [14], traditional summarization tend to identify and discard redundancies, while in sentiment-laden text, similar opinions mentioned multiple times across documents are crucial indicators of the overall strength of the sentiments expressed by the writers.

More specifically, sentiment-laden documents like product and service reviews are usually either about a single entity, e.g., consumers' goods such as digital cameras, DVD players, books; or related to user's experiences with a service like when lodging in an hotel or dining in a restaurant. An entity has several ratable features or aspects which may be targeted by reviewers with their positive or negative opinions. In this sense, each review can be viewed as 
a set of aspects with associated opinions. Ratings define the strength and the polarity of the opinions and typically range over integer values often visualized with star symbols. When summarizing reviews, it is fundamental to identify the opinion information expressed across the reviews and their polarity distribution, so that the sentences selected by a summarizer could be representative of the overall sentiment distribution. In addition to the traditional tasks, a multi-document opinion-oriented summarizer requires an information extractions stage to identify topics and polarity described in the documents.

In the restaurant domain case, many web sites $^{1}$ allow reviewers to directly rate pre-defined aspects: atmosphere, food, value, service, and overall with ratings ranging from poor (one star) to excellent (five stars). These rated aspects quantify opinions and polarities expressed in each review by the reviewers, and although there might be inconsistencies, it is safe to assume that the text document associated with the ratings carries the same sentiment contributions quantified by the number of stars. By the same token, aggregating the ratings of the single reviews over the aspects could fairly represent a summary of the overall sentiments expressed by the reviewers the specific restaurant or reviewed entity. Based on this considerations, we can assume that a summary should convey the same distribution of ratings over aspects obtained by combining the rating contribution of each review, so that each opinion contribution, even if controversial, should be represented into the final summary.

This work proposes STARLET, a novel approach to summarization of evaluative text that leverages aspects and ratings described in the reviews as features for the summarization process. In the restaurant domain, STARLET uses atmosphere, food, value, service, and overall aspects to score each sentence in the input documents. For each aspect, STARLET computes a rating indicating how much the current sentence has contributed to that aspect. For this STARLET uses a maximum entropy rating model. The predicted aspect ratings are used in a summarization model to (1) compute a score for each sentence and (2) to derive a summary score. The model is a linear weighted model with aspects as features and associated weights learned using $A^{*}$ search and discriminative training [15].

The rest of this paper is structured as follows: Section II reports current contributions to summarization of evaluative text. Section III describes the summarization model, while Section IV outlines the weight learning procedure. Section $\mathrm{V}$ illustrates the aspect rating prediction model, which is followed by a description of the experimental setup in Section VI. We presents the results in Section VII and conclude with Section VIII.

\footnotetext{
${ }^{1}$ we8there.com, tripadvisor.com, citysearch.com
}

\section{BACKGROUND}

From a high level point of view, approaches to multidocument summarization can be divided into extractive or abstractive. Extractive summarization assumes that fragments (phrases or sentences) extracted from the original documents can be used to coherently assemble a shorter version of the original text without substantially changing the information conveyed by the source. Abstractive summarization generates new documents by analyzing the semantic content of the original documents and using natural language generation techniques. While both types of summarization have been extensively studied for factual and edited text documents, evaluative text summarization has focused mostly on sentiment analysis and information extraction.

Features or aspects extraction, for instance, has been explored from many angles: topic models [16], [17], NLPbased information extraction [18], [19], semi-supervised and supervised machine learning techniques [20]. Similarly, for polarity strength prediction (NLP-based [21]) and multiaspect multi-rating prediction (regression and classification models [22], [23], [24]) are used. Most of the evaluative text summarization methods try to organize the sentimentladen sentences according to aspect and polarity. In Blair et al. [25], sentences are qualitatively aggregated by aspect and "star ratings" based on a manually-defined strategy ; $\mathrm{Hu}$ and Liu [7] simply lists the sentences by aspects and polarity; in [18], [26] aspect and polarities are graphically organized and visualized. None of these approaches considers text summaries in terms of rating distributions neither introduce metrics to quantitatively evaluate the quality of the summary. To our knowledge, the most complete contribution to evaluative text summarization are described in Carenini et al. [27] and it closely relates to this work.

In [27], Carenini et al. compare an extractive summarization system, MEAD* - a modified version of the open source summarization system MEAD [28] - with SEA, an abstractive summarization system, demonstrating that both systems perform equally well. We only consider extractive summarization.

As noticed in [27], none of sentence extraction and ranking techniques used in MEAD take into account the sentiment-laden content present in the source documents. Carenini et al. observed that the most informative sentences are the one with the highest number of crude features $(\mathrm{CF})$, where $\mathrm{CF}$ are defined as the rated aspects of the entity evaluated in the reviews. For each sentence $s_{k}$, this score is quantified by the following summation:

$$
C F_{\text {sum }}\left(s_{k}\right)=\sum_{p s_{i} \in \operatorname{eval}\left(s_{k}\right)}\left|p s_{i}\right|
$$

Where $\operatorname{eval}\left(s_{k}\right)$ is the set of crude features with sentiment-laden content in the sentence, and $\left|p s_{i}\right|$ is the absolute value of the polarity of the crude features referred 
to in the sentence. For instance, crude features for a digital camera may include battery life, zoom, picture quality, etc. If the sentence $k$ mentions positively $(+3)$ the zoom and negatively $(-2)$ the picture quality, the crude feature score would be: $C F_{\text {sum }}\left(s_{k}\right)=|+3|+|-2|=5$. CF features are used in a modified version of MEAD, called MEAD*, to rescore sentences in the final stage of content selection. For this purpose, sentences are inserted and sorted by score in $\mathrm{CF}$ 'buckets'. From the CF 'buckets' with more items, MEAD* extracts the sentence with the highest score and removes it from the pool to avoid redundancy. In case two sentences have the same score, the centroid-based feature from MEAD is used as a 'tie-breaker'. The selected sentences are finally ordered according to a pre-defined taxonomy of features where a depth-first traversal of the hierarchy makes sure that aspects are ordered from general to specific aspects.

This approach, although better than the traditional MEAD, presents a few drawbacks. Firstly, the sentence selection mechanism only considers the most frequently discussed aspects, leaving to the maximum summary lengths parameter the decision about where to stop the selection process. This could leave out interesting opinions that are not appearing sufficiently frequent in the source documents. Ideally, all the opinions should be represented in the summary according to the overall distribution of the input reviews. Secondly, using the absolute value when calculating $C F_{\text {sum }}\left(s_{k}\right)$ flattens the opinion distribution since sentences either with very negative polarity, very positive, or with numerous opinions, but with moderate polarity strengths, would get the same score, regardless. Finally, how to use the summarization features is established a priori based on expert knowledge and prior work in this area, while how to weight these features should be learned from data using automatic quality metrics. In the next few sections, we will address some of these limitations.

\section{SUMMARIZATION MODEL}

Our summarization model $s$ is an adaption of the one described in Aker et al. [15]. With this model, each possible text summary can be scored as a weighted sum of features according to the equation below:

$$
s(\mathbf{y} \mid \mathbf{x})=\Phi(\mathbf{y} \mid \mathbf{x}) \cdot \boldsymbol{\Lambda}
$$

Where $\mathbf{x}$ is a vector of indexes representing the $N$ sentences in the document set to summarize, $\mathbf{y} \subseteq\{1, \ldots, N\}$ is the set of indexes selected for the summary of length $|\mathbf{y}|=M, \boldsymbol{\Lambda}=\left\{\lambda_{1}, \ldots, \lambda_{F}\right\}$ is the weight vector parameters for the $F$ features that optimizes the summary evaluation metrics, and $\Phi(\cdot \mid \cdot)$ is a function that returns a set of features for each candidate summary. We assume that all our features can be determined independently ${ }^{2}$ leading us to

\footnotetext{
${ }^{2}$ This assumption does not take in consideration global features such as redundancy or coherence, but it can be easily extended to remove the limitation.
}

the following score function which is now only depended on a linear combination feature functions:

$$
s(\mathbf{y} \mid \mathbf{x})=\sum_{i \in \mathbf{y}} \phi\left(x_{i}\right) \lambda_{i}
$$

We can finally formulate the summarization task as search problem, where the optimal summary is the one that maximizes the following arg max decision rule:

$$
\hat{\mathbf{y}}=\underset{\mathbf{y}}{\arg \max } s(\mathbf{y} \mid \mathbf{x})
$$

The challenge is to find the scoring parameters $\boldsymbol{\Lambda}$ that produce a summary $\hat{\mathbf{y}}$ that best approximates an ideal summary $\mathbf{y}$, when compared to a gold standard reference summary using an automatic evaluation metric.

\section{Feature Weight LEARNing}

An extractive multi-document summary can be created by traversing a directed acyclic graph where each node $i$ represents a particular summary of length $l(i)$ composed by a set of sentences $S_{i}$, and a set of edges $(i, j)$. Traversing the edge $(i, j)$ incrementally adds a sentence from the set of available sentences to the previous sentence set $S_{i}$. Figure 1 shows a graphical representation of the process of selecting sentences. Each node in the graph can be evaluated by a scoring function which quantifies how good the node is when compared to a target node. In order to find the best scoring summary with a specific word length $W$, it would be necessary to search an exponentially large space with complexity $\mathcal{O}\left(S^{L(W)}\right)$ where $S$ is the total number of sentences to search and $L(W)$ is the number of sentences that best matches the required summary word length $W$.

An A* search algorithm can be used to efficiently traverse the graph and accurately find the optimal path. It applies a best-first strategy to traverse the graph from the initial node (summary of length zero) to the final node (summary of length $W$ ), and uses a heuristic function to determine the order of the nodes to explore first. The search algorithm is guaranteed to converge to the optimal solution if the heuristic function is monotonic or follows the admissible heuristic requirements. That is, the estimating path cost function from the current node to the goal never overestimates the actual cost. We used the "final aggregated heuristic" function described in [15] that satisfies the admissible heuristic constraints. The input to the heuristic is the set of sentences sorted according to their scores. ${ }^{3}$ The heuristic first adds the highest scoring sentence into the summary. After adding a sentence, the summary length is updated. If the length limit of the summary is not violated then the next highest scoring sentence is added. When the next high scoring sentence is too long to be added to the summary the heuristic

\footnotetext{
${ }^{3}$ Sentence scores are computed by the weighted linear combination of their features.
} 


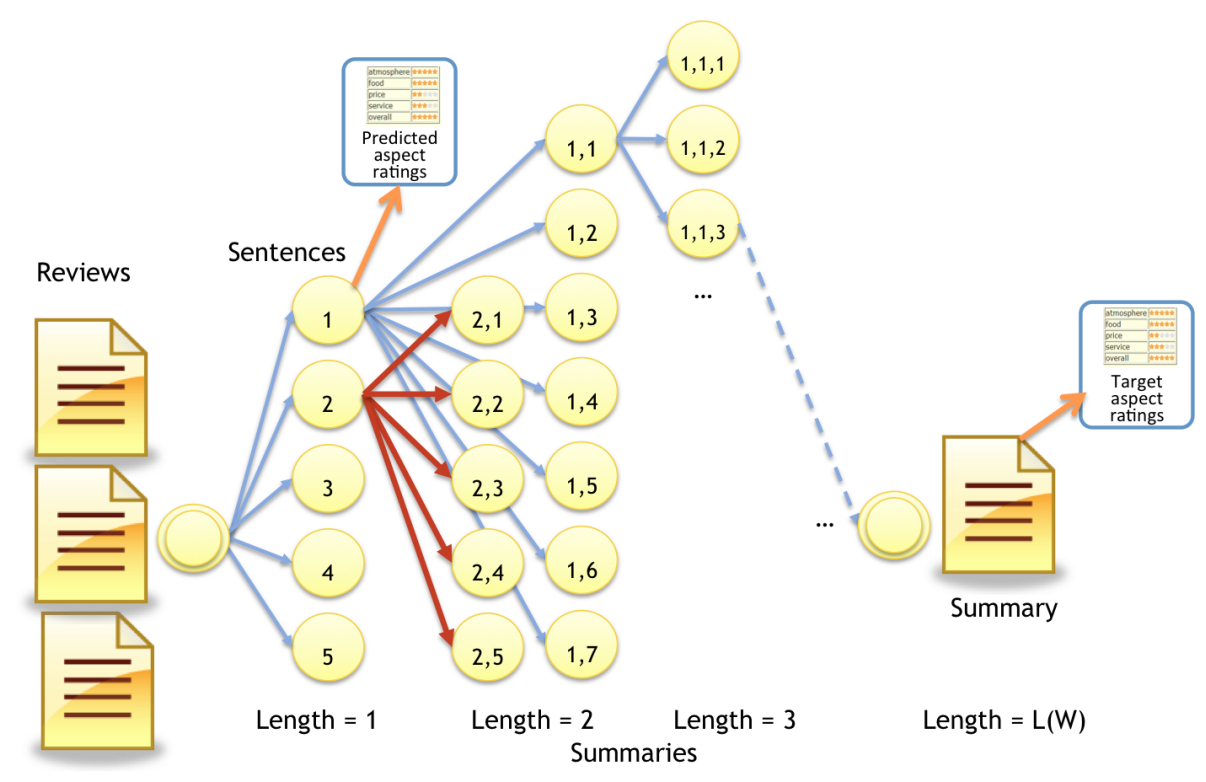

Figure 1. Creating an extractive summary by traversing a directed acyclic graph

skips this and continues with the next one until it finds the best scoring sentence that does fit into the summary. We generated summaries with a 100 -word limit based on the average length of summaries in the reference data.

\section{A. Discriminative Training}

After a summary is generated by $\mathrm{A}^{*}$ search, it is compared to a human summary using an evaluation metric such as ROUGE [29]. To learn the feature weights we used the training set (see Section VI-A) and generate for each topic a list of candidate summaries (100 summaries were created for each topic). The summaries along with their feature values and ROUGE scores are input to a MERT (Minimum Error Rate Training) module to train the weights. MERT is a first order optimization method using Powell search to find the parameters which minimize the loss on the training data [30]. It is commonly used for training statistical machine translation systems.

\section{Feature Extraction: Ratings Prediction MODEL}

In order to apply the search techniques described above, it is necessary to define a set of features relevant to the summarization task that can be determined at each step of the search process. As previously mentioned, reviews refer to specific aspects or topics of the target entity. For instance, reviews about restaurants will address opinions about the quality of the food, the courtesy of the wait personnel, or the ambience. These aspects are typically rated with certain number of stars ranging from one (poor) to five (excellent). Based on [22], it is possible to create a rating prediction model that, for each topic $t_{i} \in \mathcal{T}$, (e.g., $\mathcal{T}=\{$ food, service, ambience, value, overall $\}$ ), estimates the ratings $r_{i}$ for any review document $d_{j}$ in the considered domain $d_{j} \in \mathcal{D}$, as:

$$
\begin{aligned}
\hat{r_{i}} & =\underset{r \in \mathcal{R}}{\arg \max } P\left(r_{i} \mid d_{j}\right) \\
& =\underset{r \in \mathcal{R}}{\arg \max } P\left(r_{i} \mid s_{1, j}, s_{2, j}, \ldots, s_{n, j}\right)
\end{aligned}
$$

where each document $d_{j}$ is composed by $n$ sentences or phrases $s_{1, j}, s_{2, j}, \ldots, s_{n, j}$. We used a maximum entropy (MaxEnt) [31] model to estimate the conditional probability of the ratings (Eq. 6) given the features extracted from the text documents.

Figure 2 shows the architecture of our rating prediction model system. In this configuration, each review document is associated to a set of predefined aspects that have been assessed by the reviewers with star-rating evaluations. During training, text features such as n-grams, part of speech, shallow parsing chunks and others are used together with the reviewer's-assigned ratings to create a discriminative model classifier. There are five classifiers, one for each aspect.

For each document, the MaxEnt models will produce an estimate of the rating probability distributions $\hat{r_{i}}$, describing how ratings, for a specific aspect, are likely to be associated to the document text. For instance, a review like the following: The service was flawless timely and non intrusive. Everything was great. would have a rating distribution for the aspects service and overall skewed toward the high ratings (four or five) and almost uniform rating distribution for the remaining aspects. 


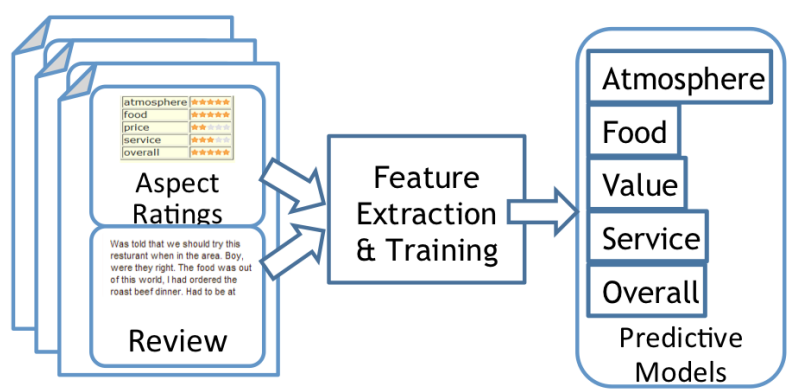

Figure 2. Rating predictive models

The rating prediction models are trained from 6,823 restaurant reviews using the approach described in [22]. Performances measured in terms of rank loss average 0.63 across all the aspects.

The process starts by calculating a set of feature scores for the initial candidate sentences, e.g., for each sentence and each aspect, it calculates the KL-divergence [32] between the predicted sentence rating distribution (as provided by MaxEnt) and a target rating distribution. This will quantify how far is the current distribution from the target. For each restaurant in the training set, we create a reference rating distribution by counting the rating contributions for each aspect in every review. The assumption is that the target text summary for a restaurant should reflect the same reference rating distribution calculated on the whole reviews set, but with much less words. In other terms, the sentences should be selected based on their contribution toward the target rating distribution. The iterative process continues as described in Section IV till the MERT weights converge. Once the training ends, the optimal $\lambda$ coefficients are used to in the $\mathrm{A}^{*}$ search heuristic to traverse the graph and generate the optimal summary from the test set.

\section{EXPERIMENTAL SETUP}

\section{A. Data}

The set of review documents used in our experiments were selected from a corpus of previously mined restaurant reviews from the we8there.com web site. In addition to the textual data, we8there.com provides numerical ratings for five predefined aspects: atmosphere, food, value, service, and overall. From the set of 3,866 available restaurants, we selected 131 with more than five reviews. Then, we manually searched for extra reviews on other web sites and selected 60 of the 131 restaurants that had reviews highly voted by web readers as useful. For each of the 60 restaurants, we selected the review with the highest number of "helpful votes" that was dated in the same time frame as the we8there.com reviews and use it as a reference summary. We randomly split the 60 restaurants into 40 for training and 20 for testing. Tables I and II gives info about the two data sets.
Table I

TEST DATA SET (20 RESTAURANTS) VALUES PER DOCUMENT SET

\begin{tabular}{|c|c|c|c|c|}
\hline \hline & Min & Max & Avg & Total \\
\hline \hline Reviews & 6 & 10 & 7.55 & 151 \\
\hline Sentences & 15 & 140 & 54.4 & 1,088 \\
\hline Words & 206 & 2,042 & 809.85 & 16,197 \\
\hline \hline
\end{tabular}

Table II

Train Data SET (40 RESTAURANTS) VALUES PER DOCUMENT SET

\begin{tabular}{|c|c|c|c|c|}
\hline \hline & Min & Max & Avg & Total \\
\hline \hline Reviews & 6 & 10 & 7.5 & 300 \\
\hline Sentences & 15 & 108 & 51.95 & 2,078 \\
\hline Words & 205 & 1,902 & 789.95 & 31,598 \\
\hline \hline
\end{tabular}

\section{B. Entire Process}

Our summarization process starts by calculating a set of features for every sentence in the training and testing set as described in Section V. In this experiments, the features we optimized with regard to the ROUGE score are the KL-divergence measure between the target aspect rating distribution and the predicted rating distributions calculated on the input sentences. The assumption is that the resulting summary will cover the target sentiments expressed in term of star-ratings by selecting the content that closer mimic the desired distribution and, at the same time, remains within the maximum summary size (100 words). In the next step, the features are weighted and their summation is used to score each sentence. The weights are trained using the training set and the algorithms described in Section IV. Finally, the trained weights are used in the scoring functions used by A* search to generate summaries for the test set.

\section{RESUlts}

To evaluate our STARLET approach, we compared it with two summarizers: 1) a baseline summarization system that randomly selects sentences with no repetition till it reaches the desired length of 100 words; 2 ) the open source MEAD system with the same output length. The resulting summaries were assessed using the automatic metric ROUGE and manual evaluation. Examples summaries are shown in Table III.

\section{A. ROUGE Evaluation}

ROUGE is a well-known evaluation method for summarization, which is based on the common number of ngrams between a peer, and one or several model summaries. The metrics taken into consideration for this evaluation are ROUGE-1 (R-1), ROUGE-2 (R-2) and ROUGE-SU4 (RSU4). R-1 and R-2 compute the number of unigrams and bigrams, respectively, that coincides in the automatic and model summaries. R-SU4 measures the overlap of skipbigrams between them allowing a skip distance of 4 . 


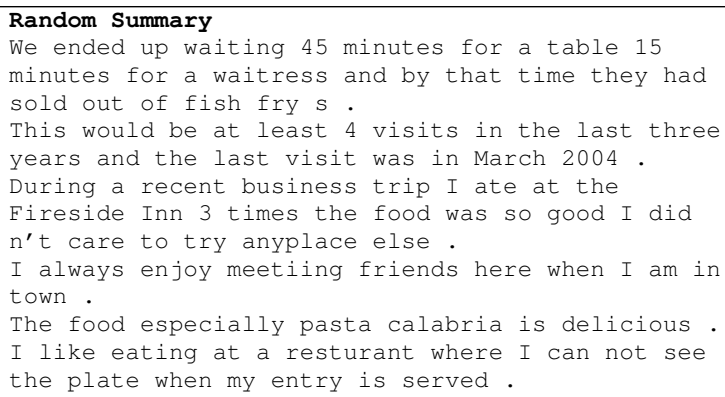

Table III

EXAMPLE OF SUMMARIES EXTRACTED FROM A SET OF 10 RESTAURANT REVIEWS

Table IV

ROUGE SCORES OBTAINED FROM THE TEST SET

\begin{tabular}{|c|c|c|c|}
\hline \hline Metric & Random & MEAD & STARLET \\
\hline \hline R-1 & 0.2769 & 0.2603 & 0.2894 \\
\hline R-2 & 0.0329 & 0.0377 & 0.0454 \\
\hline R-SU4 & 0.0790 & 0.0727 & 0.0881 \\
\hline \hline
\end{tabular}

From Table IV we can see that STARLET outperforms the other two systems in all ROUGE metrics. This means that, according to ROUGE, our summarizer generates reviews/summaries whose lexical content is closer to human ones and thus are more likely to capture the opinions about the restaurant than the other two systems.

\section{B. Manual Evaluation}

In the manual evaluation we asked three people (two of them native speakers of English) to evaluate the readability of the generated summaries according to the evaluation criteria described in [33]. Without showing the reference
Table V

MANUAL EVALUATION FOR THE THREE SUMMARIZATION SYSTEMS

\begin{tabular}{|c|c|c|c|}
\hline \hline & Random & MEAD & Starlet \\
\hline \hline Grammatically & 3.53 & 3.68 & 3.67 \\
\hline Redundancy & 2.82 & 2.92 & 3.00 \\
\hline Clarity & 2.78 & 2.97 & 3.05 \\
\hline Coverage & 2.67 & 2.33 & 3.23 \\
\hline Coherence & 2.05 & 2.57 & 2.62 \\
\hline \multicolumn{4}{|l}{} \\
\hline
\end{tabular}

summary, we asked each participant to rate the following linguistic qualities with a rating scale ranging from a maximum of 5 (very good) to a minimum of 1 (very poor): Grammaticality - grammatically correct and without artifacts; Redundancy - absence of unnecessary repetitions; Clarity - easy to read; Coherence - well structured and organized. Since the Focus readability property listed in [33] applies mostly to the DUC summarization tasks, we replaced it with Coverage that indicated level of coverage for the aspects and the polarity expressed in the summary. In other terms, this rate should be higher if most of the sentences are expressing opinions on the pre-defined restaurant aspects. The average scores for each criterion are shown in Table V.

From Table V we can see that the scores for Grammaticality, Redundancy, Clarity and Coherence are in all systems very close to each other. The only gap can be observed in the Coverage metric. This metric expresses how many opinions and aspects are actually covered in the review/summary. The scores indicate that STARLET is able to generate summaries with a wider range of opinions than the other two systems.

\section{Discussion}

Reviews are typically written by consumers to convey their personal opinion of a product or a service. Compared to traditional automatic summarization tasks - where the documents are usually written by professionals, edited, and proofread - the English quality of reviews is usually poor, often ungrammatical, incoherent, and inconsistent. While in news-based documents, facts and events are the central topics expressed by the author, reviews are focused on attributes or features of a product and the reviewer's opinions about the qualities of such product characteristics.

Looking at the manual evaluation from the judges, the 'grammatically' scores are consistent across the three methods and depend only on the quality of the source sentence. Poorly written sentences can be penalized by introducing new features during training that take into consideration the number of misspellings (for instance, in our data, the word atmosphere has been misspelled in 23 different ways), the number of words belonging to the English dictionary, and scores from a parser.

The 'redundancy' score is slightly better for STARLET, but in the current version there is not a mechanism to avoid similar sentences, although selecting sentences according to the rating distribution should help to reduce redundancy. 
Sentence similarity features can be added during training by using centroid-based clustering and demote similar sentences to these already included in the summary.

Also the 'clarity' and 'coherence' scores are better in our approach. Low scores are related to controversial reviews where the opinions are mixed and distributed across the ratings. In these cases, more investigation is necessary, perhaps ordering positive and negative sentences according to some rhetorical structure or learned-from-data language models.

Finally, the 'coverage' score for STARLET is decidedly better than for the other approaches, showing that STARLET correctly selects information relevant to the users.

\section{CONClusions AND FUtURE WORK}

This paper addresses extractive summarization for reviews containing opinions on multiple aspects of the entity object being reviewed. We propose a method called STARLET. It uses aspects as features to score sentences in the input documents. The features are weighted linearly and summaries are generated using $\mathrm{A}^{*}$ search. We trained the weights using MERT and use "best" reviews as golden standard summaries. We performed both automatic and manual evaluations in the restaurant reviews domain. In both evaluations the results show that STARLET summaries contain more review information than alternative baselines.

In future work we plan to study the integration of other aspects such as redundancy and coherence into the feature weight learning process. In addition to this we plan to investigate more appropriate evaluation metrics for review summaries and explore more features as described in the discussion section.

\section{ACKNOWLEDGMENT}

The authors would like to thank Amanda Stent, Alistair Conkie, Patrick Haffner, Narendra Gupta, and the anonymous reviewers who provided useful feedback and suggestions.

\section{REFERENCES}

[1] W. Duan, B. Gu, and A. B. Whinston, "Do Online Reviews Matter? - An Empirical Investigation of Panel Data," Journal Decision Support Systems, vol. 45, no. 4, pp. 1007-1016, November 2008.

[2] D. Park, J. Lee, and I. Han, "The effect of on-line consumer reviews on consumer purchasing intention: The moderating role of involvement," Int. J. Electron. Commerce, vol. 11, pp. 125-148, July 2007.

[3] J. Feng, M. Johnston, and S. Bangalore, "Speech and multimodal interaction in mobile search," Signal Processing Magazine, IEEE, vol. 28, no. 4, pp. 40 -49, july 2011.
[4] A. Stent, I. Zeljković, D. Caseiro, and J. Wilpon, "Geocentric language models for local business voice search," in Proceedings of Human Language Technologies: The 2009 Annual Conference of the North American Chapter of the Association for Computational Linguistics, ser. NAACL '09. Stroudsburg, PA, USA: Association for Computational Linguistics, 2009, pp. 389-396.

[5] B. Pang and L. Lee, "Opinion mining and sentiment analysis," Foundations and Trends in Information Retrieval, vol. 2, no. 1-2, pp. 1-135, 2008.

[6] R. McDonald, K. Hannan, T. Neylon, M. Wells, and J. Reynar, "Structured models for fine-to-coarse sentiment analysis," in Proceedings of the Association for Computational Linguistics $(A C L)$. Prague, Czech Republic: Association for Computational Linguistics, June 2007, pp. 432-439.

[7] M. Hu and B. Liu, "Mining and summarizing customer reviews," in Proceedings of the ACM SIGKDD Conference on Knowledge Discovery and Data Mining (KDD), 2004, pp. $168-177$.

[8] J. M. Conroy, J. G. Stewart, and J. D. Schlesinger, "CLASSY query-based multi-document summarization," in In Proceedings of the Document Understanding Conf. Wksp. 2005 (DUC 2005) at the Human Language Technology Conf./Conf. on Empirical Methods in Natural Language Processing (HLT/EMNLP, 2005.

[9] K. R. McKeown, R. Barzilay, D. Evans, V. Hatzivassiloglou, J. L. Klavans, A. Nenkova, C. Sable, B. Schiffman, and S. Sigelman, "Tracking and summarizing news on a daily basis with columbia's newsblaster," in Proceedings of the second international conference on Human Language Technology Research, ser. HLT '02. San Francisco, CA, USA: Morgan Kaufmann Publishers Inc., 2002, pp. 280-285.

[10] N. Elhadad, M. Y. Kan, J. L. Klavans, and K. R. McKeown, "Customization in a unified framework for summarizing medical literature," Artif. Intell. Med., vol. 33, pp. 179-198, February 2005.

[11] T. Copeck, N. Japkowicz, and S. Szpakowicz, "Text summarization as controlled search," in Proceedings of the 15th Conference of the Canadian Society for Computational Studies of Intelligence on Advances in Artificial Intelligence, ser. AI '02. London, UK, UK: Springer-Verlag, 2002, pp. 268280.

[12] H. Saggion and L. Guy, "Generating indicative-informative summaries with sumum," Comput. Linguist., vol. 28, pp. 497526, December 2002.

[13] S. Mithun and L. Kosseim, "Summarizing blog entries versus news texts," in Proceedings of the Workshop on Events in Emerging Text Types, ser. eETTs '09. Stroudsburg, PA, USA: Association for Computational Linguistics, 2009, pp. 1-8.

[14] L. W. Ku, Y. T. Liang, and H. H. Chen, "Opinion extraction, summarization and tracking in news and blog corpora," in Proceedings of AAAI-2006 Spring Symposium on Computational Approaches to Analyzing Weblogs, 2006. 
[15] A. Aker, T. Cohn, and R. Gaizauskas, "Multi-document summarization using $\mathrm{a}^{*}$ search and discriminative training," in Proceedings of the 2010 Conference on Empirical Methods in Natural Language Processing. Association for Computational Linguistics, 2010, pp. 482-491.

[16] I. Titov and R. McDonald, "A joint model of text and aspect ratings for sentiment summarization," in Proceedings of ACL08: HLT, 2008, pp. 308-316.

[17] Q. Mei, X. Ling, M. Wondra, H. Su, and C. Zhai, "Topic sentiment mixture: modeling facets and opinions in weblogs," in WWW '07: Proceedings of the 16th international conference on World Wide Web. ACM, 2007, pp. 171-180.

[18] B. Liu, M. Hu, and J. Cheng, "Opinion observer: analyzing and comparing opinions on the web," in Proceedings of the 14th international conference on World Wide Web, ser. WWW '05. New York, NY, USA: ACM, 2005, pp. 342-351.

[19] A.-M. Popescu, B. Nguyen, and O. Etzioni, "OPINE: Extracting product features and opinions from reviews," in Proceedings of HLT/EMNLP 2005 Interactive Demonstrations. Association for Computational Linguistics, 2005, pp. 32-33.

[20] G. Qiu, B. Liu, J. Bu, and C. Chen, "Opinion word expansion and target extraction through double propagation," Comput. Linguist., vol. 37, pp. 9-27, 2011.

[21] A. Esuli, "Automatic generation of lexical resources for opinion mining: models, algorithms and applications," SIGIR Forum, vol. 42, pp. 105-106, November 2008.

[22] N. Gupta, G. Di Fabbrizio, and P. Haffner, "Capturing the stars: predicting ratings for service and product reviews," in Proceedings of the NAACL HLT 2010 Workshop on Semantic Search, ser. SS '10. Stroudsburg, PA, USA: Association for Computational Linguistics, 2010, pp. 36-43.

[23] B. Snyder and R. Barzilay, "Multiple aspect ranking using the good grief algorithm," in Human Language Technologies 2007: The Conference of the North American Chapter of the Association for Computational Linguistics; Proceedings of the Main Conference. Association for Computational Linguistics, 2007, pp. 300-307.

[24] S. Baccianella, A. Esuli, and F. Sebastiani, "Multi-facet rating of product reviews," in Proceedings of the 31th European Conference on IR Research on Advances in Information Retrieval, ser. ECIR '09. Berlin, Heidelberg: SpringerVerlag, 2009, pp. 461-472.

[25] S. Blair-Goldensohn, K. Hannan, R. McDonald, T. Neylon, G. Reis, and J. Reynar, "Building a Sentiment Summarizer for Local Service Reviews," in NLPIX, 2008.

[26] G. Carenini and L. Rizoli, "A multimedia interface for facilitating comparisons of opinions," in IUI '09: Proceedings of the 13th international conference on Intelligent user interfaces. ACM, 2009, pp. 325-334.

[27] G. Carenini, R. Ng, and A. Pauls, "Multi-document summarization of evaluative text," in 11th Meeting of the European Chapter of the Association for Computational Linguistics (EACL 2006), 2006.
[28] D. Radev, T. Allison, S. Blair-Goldensohn, J. Blitzer, A. Çelebi, S. Dimitrov, E. Drabek, A. Hakim, W. Lam, D. Liu, J. Otterbacher, H. Qi, H. Saggion, S. Teufel, M. Topper, A. Winkel, and Z. Zhang, "MEAD - A platform for multidocument multilingual text summarization," in Conference on Language Resources and Evaluation (LREC), Lisbon, Portugal, May 2004.

[29] C. Lin, "Rouge: A package for automatic evaluation of summaries," in Proceedings of the workshop on text summarization branches out (WAS 2004), 2004, pp. 25-26.

[30] F. Och, "Minimum error rate training in statistical machine translation," in Proceedings of the 41st Annual Meeting on Association for Computational Linguistics-Volume 1. Association for Computational Linguistics, 2003, pp. 160-167.

[31] A. L. Berger, V. J. D. Pietra, and S. A. D. Pietra, "A maximum entropy approach to natural language processing," Comput. Linguist., vol. 22, pp. 39-71, March 1996.

[32] S. Kullback and R. A. Leibler, "On information and sufficiency," Ann. Math. Statist., vol. 22, no. 1, pp. 79-86, 1951.

[33] H. Dang, "Overview of DUC 2005," DUC 05 Workshop at HLT/EMNLP, 2005. 